\title{
Krill protein hydrolysate reduces plasma triacylglycerol level with concurrent increase in plasma bile acid level and hepatic fatty acid catabolism in high-fat fed mice
}

\author{
Marie S. Ramsvik ${ }^{1,2}$, Bodil Bjørndal ${ }^{1}$, Rita Vik ${ }^{1}$, Inge Bruheim ${ }^{2}$, Jon Skorve ${ }^{1}$, and Rolf \\ K. Berge $\mathrm{e}^{1,3 *}$ \\ ${ }^{1}$ Department of Clinical Science, University of Bergen, N-5020 Bergen, Norway; ${ }^{2}$ Olympic \\ Seafood AS, N-6080 Myrvaag, Norway; ${ }^{3}$ Department of Heart Disease, Haukeland \\ University Hospital, N-5021 Bergen, Norway
}

*Corresponding author: Rolf $\mathrm{K}$. Berge, $\mathrm{PhD},{ }^{1}$ Department of Clinical Science, University of Bergen, N-5020 Bergen, Norway

Submission date: September 10, 2013; Acceptance date: November 12, 2013; Publication date: November 18, 2013

\begin{abstract}
Background: Krill powder, consisting of both lipids and proteins, has been reported to modulate hepatic lipid catabolism in animals. Fish protein hydrolysate diets have also been reported to affect lipid metabolism and to elevate bile acid (BA) level in plasma. BA interacts with a number of nuclear receptors and thus affects a variety of signaling pathways, including very low density lipoprotein (VLDL) secretion. The aim of the present study was to investigate whether a krill protein hydrolysate $(\mathrm{KPH})$ could affect lipid and BA metabolism in mice.
\end{abstract}

Method: C57BL/6 mice were fed a high-fat $(21 \%$, w/w) diet containing $20 \%$ crude protein $(\mathrm{w} / \mathrm{w})$ as casein (control group) or KPH for 6 weeks. Lipids and fatty acid composition were measured from plasma, enzyme activity and gene expression were analyzed from liver samples, and BA was measured from plasma.

Results: The effect of dietary treatment with KPH resulted in reduced levels of plasma triacylglycerols (TAG) and non-esterified fatty acids (NEFAs). The KPH treated mice had also a marked increased plasma BA concentration. The increased plasma BA level was associated with induction of genes related to membrane canalicular exporter proteins $(A b c c 2$, $A b c b 4)$ and to BA exporters to blood (Abcc3 and Abcc4). Of note, we observed a 2-fold increased nuclear farnesoid X receptor (Fxr) mRNA levels in the liver of mice fed KPH. We also observed increased activity of the nuclear peroxiosme proliferator-activated receptor alpha (PPAR $\alpha)$ target gene carnitine plamitoyltransferase 2 (CPT-2).

Conclusion: The KPH diet showed to influence lipid and BA metabolism in high-fat fed mice. Moreover, increased mitochondrial fatty acid oxidation and elevation of BA concentration may regulate the plasma level of TAGs and NEFAs. 
Key words: Krill protein hydrolysate, triacylglycerol, fatty acids, TNF $\alpha$

\section{INTRODUCTION}

Bile acids (BA), essential components for solubilization of dietary fats, are synthesized in the liver from cholesterol. After synthesis, they are secreted across the canalicular membrane into the biliary tree and finally end up in the gall bladder. From the gall bladder the BAs are drained into the small intestine, reabsorbed by entrocytes and secreted into portal vein in order to be returned to the liver to complete the enterohepatic cycle.

BAs interact with a number of nuclear receptors to stimulate a variety of signalling pathways. One of these receptors is the nuclear receptor farnesoid $\mathrm{X}$ receptor (FXR). The main physiological role of FXR is to maintain the BA homeostasis, and activation of FXR by BAs will regulate genes encoding proteins involved in the enterohepatic cycle. As BAs are reported to reduce plasma and liver triacylglycerol (TAG) level in animals [1, 2], this implies that FXR also play a role in the control of lipoprotein metabolism and in the secretion of very low density lipoprotein (VLDL) TAG lipoprotein fractions. Further, FXR also controls plasma TAG levels by activating the nuclear peroxiosme proliferator-activated receptor alpha $(\operatorname{PPAR} \alpha)$ gene [3]. PPAR $\alpha$ is reported to induce genes encoding enzymes involved in mitochondrial and peroxiosmal fatty acid oxidation (reviewed in [4]), and studies have shown that modulation of the fatty acid oxidation capacity is important in regulation of the TAG concentrations in serum and liver (reviewed in $[5,6]$ ).

High plasma TAG concentration is a characteristic feature of the metabolic syndrome, and our recent findings suggest that the hypotriacylglyceridemic property of PPAR $\alpha$ activation is primary due to effects on TAG synthesis and on mitochondrial fatty acid oxidation. Abnormalities in insulin signaling have also been found associated with the metabolic syndrome, and interestingly, glucose metabolism also seems to be regulated by BAs and FXR activation (reviewed in [7, 8]). Furthermore, there is an increasing body of evidence that PPAR $\alpha$ does not only regulate lipid metabolism, but also the amino acid metabolism $[9,10]$.

The beneficial health effect of dietary krill intake has now been found in a limited number of studies. The hypotriacylglycerolemia has been attributed mostly to a greater proportion of n-3 polyunsaturated fatty acids (PUFAs) in the phospholipid fraction of krill oil $[11,12]$. Recently, we reported that krill powder, which contains approximately $40 \%$ protein $(\mathrm{w} / \mathrm{w})$ and $60 \%$ fat, increased liver lipid catabolism in tumor necrosis factor alpha (TNF $\alpha$ ) transgenic mice fed a high-fat diet [13]. Thus, the hypolipidemic effect of dietary krill extract could be due to the oil and/or the protein content. It has been found that a krill peptide showed blood-pressure lowering effects in animals [14], and a water-soluble extract of krill prevented TAG accumulation in adipocytes in culture via suppression of PPAR $\alpha$ activity [15].

In the present study, we investigated whether the dietary water-soluble protein krill protein hydrolysate (KPH) was able to affect hepatic lipid and BA metabolism, and to lower plasma TAG levels in mice fed a high-fat diet for 6 weeks. We found that mice fed KPH displayed increased mitochondrial fatty acid oxidation capacity and lower plasma TAG concentration. Moreover, KPH-treated mice had a 16-fold higher fasting plasma BA levels than mice fed casein.

\section{MATERIALS AND METHODS}




\section{Animals and diets}

Male C57BL/6 mice were used (Taconic, Germantown, USA). The experiments were performed in accordance with, and under the approval of, the Norwegian State Board for Biological Experiments, the Guide for the Care and Use of Laboratory Animals, and the Guidelines of the Animal Welfare Act. Mice aged between 6 and 8 weeks were random divided into a experimental $(n=6)$ and control $(n=9)$ group, and housed in pairs in open cages with constant temperature $\left(22 \pm 2^{\circ} \mathrm{C}\right)$ and humidity $(55 \pm 5 \%)$. They were exposed to a $12 \mathrm{~h}$ light-dark cycle and had unrestricted access to tap water and food. The mice were acclimatized under these conditions with standard chow for one week prior the start of the experiment. The animals weighed $27 \pm 2 \mathrm{~g}$ at baseline, and there was no statistical difference in the weight between the groups (data not shown).

Mice were fed ad libitum for 6 weeks on a high-fat control diet or a high-fat KPH supplemented diet. The diets contained 20\% (w/w) crude protein $(\mathrm{N} \mathrm{x} \mathrm{6,25)} \mathrm{in} \mathrm{the} \mathrm{form} \mathrm{of}$ bovine milk casein (Dyets Inc., Bethlehem, PA, USA) or as the water-soluble protein KPH (Olympic Seafood AS, Myrvaag, Norway) (>70\% with a molecular weight $<1000 \mathrm{Da}$ ). All diets contained $21 \%(\mathrm{w} / \mathrm{w})$ fat consisting of lard $(19 \%$, a generous gift from Ten Kate Vetten BV, Musselkanaal, Netherlands), and soy oil (2\%, Dyets Inc.). The other constituents of the diets were cornstarch, dyetrose, sucrose, fiber, AIN-93G mineral mix, AIN-93 vitamin mix, L-Cysteine, Choline bitartrate (all from Dyets Inc.), and tert-Butyl-hydroquinone (SigmaAldrich Norway AS, Oslo, Norway). The nutrition composition of the experimental diets is described in Table 1, and the amino acid composition of casein and KPH is given in Table 2.

After 6 weeks of diet treatment, the mice were anaesthetized under non-fasting conditions by inhalation of $2 \%$ isoflurane (Schering-Plough, Kent, UK). Blood was collected by aortic puncture with $7.5 \%$ EDTA and immediately chilled on ice for a minimum of 15 minutes. The samples were centrifuged and plasma was stored at $-80^{\circ} \mathrm{C}$ prior to analysis. Parts of the liver were chilled on ice and used for enzyme activities, and the rest were freezeclamped and stored at $-80^{\circ} \mathrm{C}$ until further analysis.

\section{Plasma lipids and fatty acid composition}

Plasma lipids were extracted according to Bligh and Dyer [16], evaporated under nitrogen, and redissolved in isopropanol before analysis. Lipids from plasma were then measured enzymatically on a Hitachi 917 system (Roche Diagnostics GmbH, Mannheim, Germany), using the triacylglycerol (GPO-PAP) and cholesterol (CHOD-PAP) kit from Roche Diagnostics, and the non-esterified fatty acid (NEFA) and phospholipid kit (phospholipids FS) from DiaSys (Diagnostic Laboratories GmbH, Holzheim, Germany).

Table 1. Composition of the experimental diets*

\begin{tabular}{lll}
\hline Macronutrients & Control $(\mathbf{g} / \mathbf{k g}$ diet $)$ & KPH $(\mathbf{g} / \mathbf{k g}$ diet $)$ \\
\hline Casein & 224,7 & \\
KPH & & 266,7 \\
\hline Soybean oil & 20 & 20 \\
Lard & 190 & 190 \\
\hline Cornstarch & 90 & 90 \\
Dyetrose & 132 & 132 \\
Sucrose & 100 & 100 \\
\hline
\end{tabular}




\begin{tabular}{lll}
\hline Fiber & 50 & 50 \\
\hline Micronutrients & & \\
\hline $\begin{array}{l}\text { AIN-93G-MX mineral } \\
\text { mix }\end{array}$ & 35 & 35 \\
$\begin{array}{l}\text { AIN-93VX vitamin } \\
\text { mix }\end{array}$ & 10 & 10 \\
$\begin{array}{l}\text { L-cystine } \\
\text { Choline bitartrate }\end{array}$ & 3 & 3 \\
$\begin{array}{l}\text { Tert-Butyl- } \\
\text { hydroquinone }\end{array}$ & 0,014 & 2,5 \\
\hline
\end{tabular}

$\mathrm{KPH}$, krill protein hydrolysate.

*The diets were isonitrogenous and isoenergetic, and contained $20 \mathrm{~g}$ of crude protein $/ 100 \mathrm{~g}$ of diet. KPH consisted of $75 \%$ protein and $3 \%$ fat; casein consisted of $89 \%$ protein and $0,8 \%$ fat.

Table 2. Amino acid composition in casein and krill protein hydrolysate

\begin{tabular}{|l|l|l|l|}
\hline Amino acids & AA & $\begin{array}{l}\text { Casein } \\
(\mathbf{g} / \mathbf{1 0 0} \text { g protein })\end{array}$ & $\begin{array}{l}\text { KPH } \\
(\mathbf{g} / \mathbf{1 0 0} \text { g protein })\end{array}$ \\
\hline Aspartic acid & ASP & 7,28 & 8,17 \\
\hline Glutamic acid & GLU & 22,00 & 12,02 \\
\hline Serine & SER & 5,44 & 3,45 \\
\hline Glycine & GLY & 1,80 & 5,67 \\
\hline Arginine & ARG & 3,25 & 8,29 \\
\hline Threonine & THR & 4,05 & 3,26 \\
\hline Alanine & ALA & 2,92 & 6,35 \\
\hline Proline & PRO & 10,48 & 8,03 \\
\hline Tyrosine & TYR & 4,22 & 5,36 \\
\hline Valine & VAL & 6,23 & 4,56 \\
\hline Methionine & MET & 2,58 & 2,88 \\
\hline Isoleucine & ILE & 4,85 & 4,84 \\
\hline Leucine & LEU & 9,08 & 8,20 \\
\hline Phenylalanine & PHE & 5,16 & 4,27 \\
\hline Lysine & LYS & 7,88 & 7,68 \\
\hline Histidine & HIS & 2,76 & 0,00 \\
\hline Taurine & TAU & 0,00 & 6,97 \\
\hline Methionine/Glycine & MET/GLY & 1,43 & 0,51 \\
\hline Lysine/Arginine & LYS/ARG & 2,43 & 0,93 \\
\hline KPH, krill protein & & \\
\hline
\end{tabular}

$\mathrm{KPH}$, krill protein hydrolysate.

\section{Plasma bile acid measurement}

BA from plasma were measured enzymatically on a Roche Modular P chemistry analyzer (Roche Diagnostica), using the BA kit (Total Bile Acid Assy Kit, 05471605001) from Diazyme (Diazyme Laboratories, Gregg, USA). 


\section{Hepatic enzyme activities}

The livers were homogenized and a post-nuclear fraction was prepared as described earlier [17]. The activities of carnitine palmitoyltransferase 1 (CPT-1a, EC number 2.3.1.21) and carnitine plamitoyltransferase 2 (CPT-2, EC number 2.3.1.21), acyl-CoA oxidase 1, palmitoyl (ACOX1, EC number 1.3.3.6), glycerol-3-phosphate acyltransferase (GPAT, EC number 2.3.1.15) and fatty acid synthase (FASN, EC number 2.3.1.85) were measured in the post-nuclear fraction as described by Skorve et al.[18].

\section{Hepatic gene expression}

Total cellular RNA was purified from frozen liver samples, and cDNA was produced as previously described [19]. Real-time PCR was performed with Sarstedt 384 well multiplyPCR Plates (Sarstedt Inc., Newton, NC, USA) on the following genes, using probes and primers from Applied Biosystems: ATP-binding cassette, sub-family C, member 2 (Abcc2, Mm00496899_m1), ATP-binding cassette, sub-family C, member 3 (Abcc3, Mm00551550_m1), ATP-binding cassette, sub-family C, member 4 (Abcc4, Mm01226381_m1), ATP-binding cassette, sub-family B, member $1 \mathrm{~b}$ (Abcblb, Mm00440736), ATP-binding cassette, sub-family B, member 4 (Abcb4, Mm00435630_m1), ATP-binding cassette, sub-family B, member 11 (Abcb11, Mm00445168), bile acid Coenzyme A:amino acid N-acyl transferase (Baat, Mm00476075_m1), cytochrome P450, family 7, sub-family A, polypeptide 1 (Cyp7al, Mm00484152_m1), farnesyl X receptor (Nr1H4, Mm00436419), solute carrier family 10, member 1 (Slc10a1, Mm00441421_m1) and solute carrier organic anion transporter family, member $1 \mathrm{~b} 2$ (Slcolb2, Mm00451510_m1). Four different reference genes were included: 18S (Kit-FAM-TAMRA (Reference RT-CKFT-18s)) from Eurogentec, Belgium, glyceraldehyde-3-phosphate dehydrogenase (Gapdh, Mm99999915_g1) from Applied Biosystems, TATA box binding protein (Tbp, AX-041188-00) and hypoxanthine guanine phosphoribosyl transferase (Hprt1, AX-045271-00) from Thermo Fisher Scientific. Data normalized to $18 S$ rRNA are presented.

\section{Statistical analysis}

Data sets were analyzed using Prism Software (Graph-Pad Software, version 6, San Diego, CA) to determine statistical significance. The results are reported as means per group with their standard deviations (SD). Due to the low number of observations in each group, nonparametric statistical test were used. Mann-Whitney test was performed to evaluate statistical differences between groups, and Wilcoxon signed-rank test was performed when comparing matched pairs. $P$-values $<0.05$ were considered significant.

\section{RESULTS}

\section{Growth of mice fed krill protein hydrolysate}

The average body weight in mice fed KPH decreased slightly over the test period $(-2,3 \pm 2,3$ $\mathrm{g}$, non significant.), whereas the mice fed casein gained weight $(8,2 \pm 4,0 \mathrm{~g}, p=0,004)$, resulting in a significant difference in change in weight between the groups over the period ( $p$ $=0,0004$ ) (Fig. 1a-c). The feed consumption was significantly higher in the KPH group relative to the casein fed mice ( $p=0,01$ ) (Fig.1d). Further, the liver:body weight index was significantly lower in the KPH mice than in control $(4,1 \pm 0,4 \%$ in $\mathrm{KPH}$ vs. $4,7 \pm 0,4 \%$ in control, $p=0,01$ ) (Fig.1e). 
a

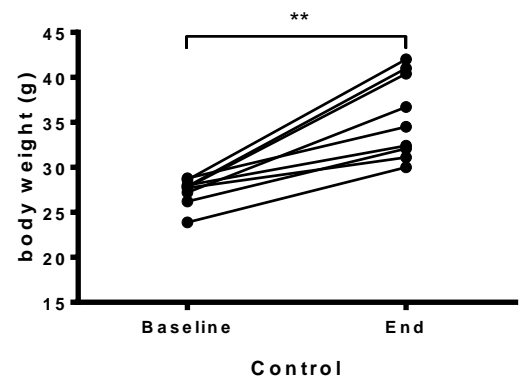

C
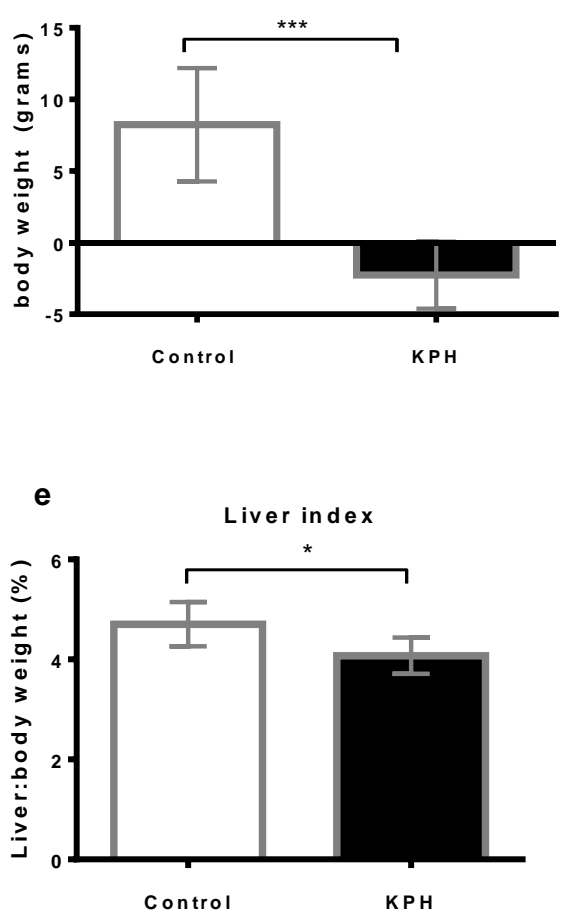

b

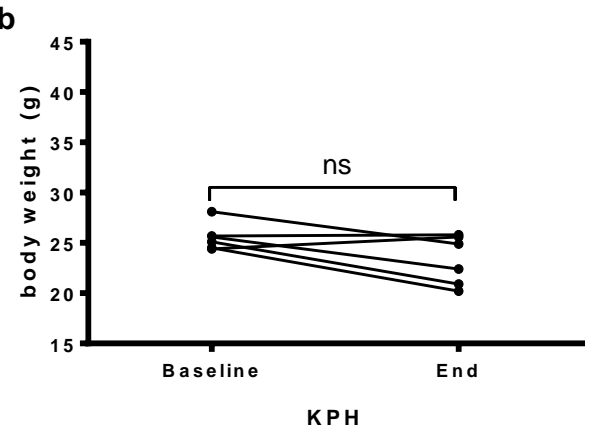

d

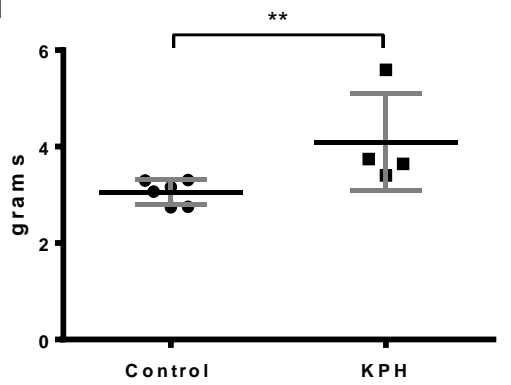

Figure 1. Body weight gain are reduced in mice fed a krill protein hydrolysate-based diet Body weight gain in (a) the krill protein hydrolysate (KPH) group compared to (c) control (b) in male C57BL/6 mice. Food intake was measured as average intake between day 5-7, and day 17-19 (d). The liver:body weight index (e) after six week of high-fat feeding containing casein or KPH. $n=6$ for KPH groups and $n=9$ for control groups, except for measurements of food intake (d), in which $n=4$ for KPH group and $n=6$ for control. Data in (c-e) are presented as mean $\pm \mathrm{SD}$. $*$ Denote significant difference: Mann-Whitney test; Wilcoxon signed-rank test $(*=p<0,05 ; * *=p<0,01 ; * * *=p<0,001)$. KPH, krill protein hydrolysate; ns, nonsignificant.

\section{Reduced plasma lipids by feeding krill protein hydrolysate}

The fasting plasma levels of TAGs, phospholipids and NEFAs were significantly reduced in the KPH treated mice compared to control ( $p=0,0007 ; p=0,03 ; p=0,03$, respectively) (Fig. 2a-c). No difference was found in total plasma cholesterol (Fig. 2d).

\section{Hepatic enzyme activities}

Plasma TAG levels are determined by a balance between hepatic TAG synthesis and secretion on one hand, and plasma TAG clearance on the other. Moreover, the hepatic fatty acid oxidation capacity will regulate the VLDL synthesis and secretion. Interestingly, we found that the activity of PPAR- $\alpha$ targeted gene CPT- 2 was stimulated in KPH fed mice 
compared to control ( $\mathrm{p}=0,015$ ) (Fig. 3a). Higher activity of other PPAR- $\alpha$ targeted genes, $\operatorname{ACOX} 1(\mathrm{p}=0,09)$ (Fig. 3b) and CPT-1 (in both absence and presence of malonyl-CoA), was also found in KPH relative to casein fed mice, but the differences did not reach statistical significance (CPT-1 with malonyl-CoA, $p=0,07$; without malonyl-CoA, $p=0,15$ ) (Fig. 3c). Hepatic TAG biosynthesis and lipogenesis seemed to be unaffected by KPH treatment as the activities of GPAT and FAS were similar in KPH treated mice compared to controls (Fig. 3d, e).
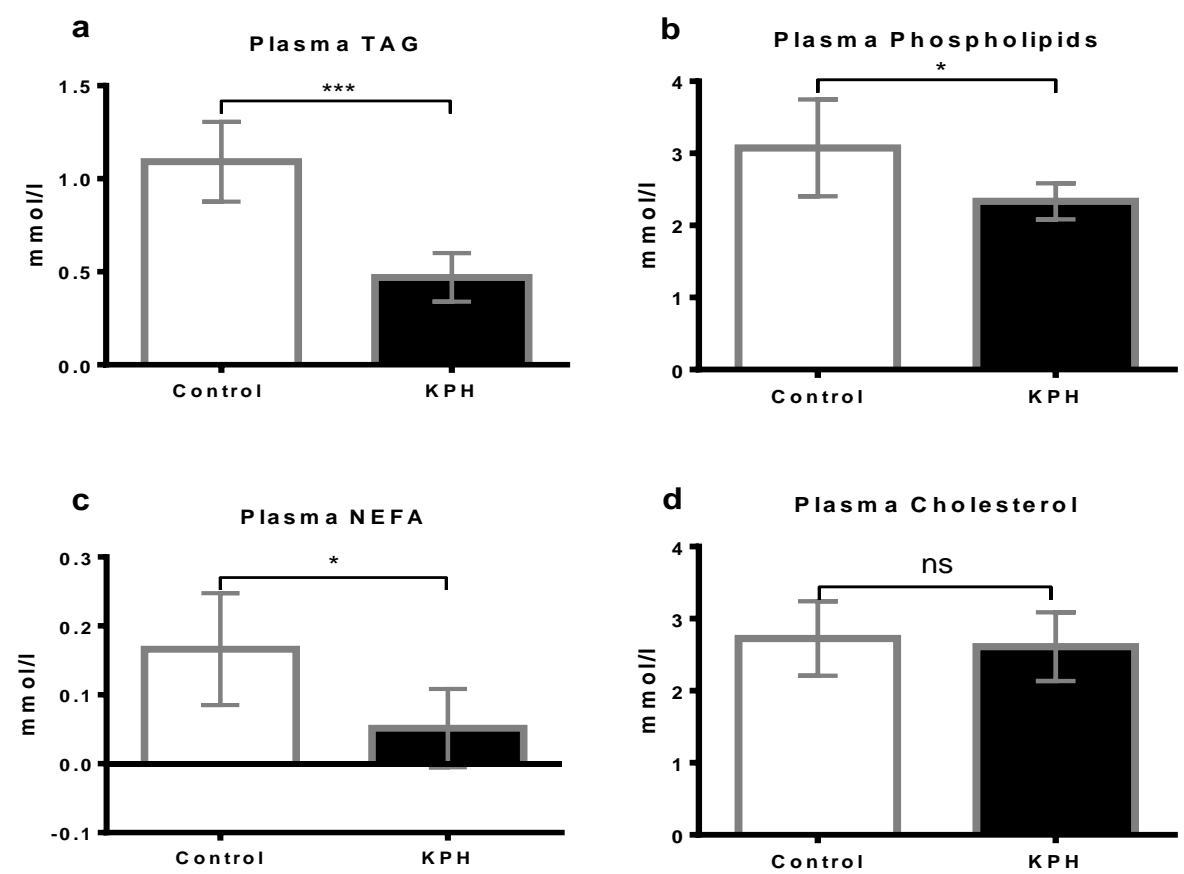

Figure 2. Krill protein hydrolysate reduces fasting plasma triacylglycerol

Concentrations of (a) triacylglycerol (TAG), (b) phospholipids, (c) non-esterified fatty acids (NEFA) and (d) cholesterol in plasma of fasted male C57BL/6 mice fed high-fat diets containing casein or KPH. Data are presented as mean $\pm \mathrm{SD}, n=6$ for KPH groups and $n=8$ for control groups. * Denote significant difference: Mann-Whitney test $(*=p<0,05 ; * * *=p<0,001) . K P H$, krill protein hydrolysate; $n s$, non-significant; TAG, triacylglycerol; NEFA, non-esterified fatty acid.

\section{Plasma bile acid and hepatic genes of the enterohepatic cycle}

It is reported that BAs have the potential to reduce plasma TAG levels $[1,2,8,20]$. Therefore, it was of interest to note that KPH treatment increased the plasma BA level a 16fold compared to mice fed casein (Fig. 4).

BAs are synthesized in the liver from cholesterol. The expression of the rate-limiting enzyme in this synthesis, Cyp7al, was up-regulated in the liver of mice fed KPH. However, it did not reach statistical significance $(p=0,06)$ (Fig. 5a). After BA is synthesized, it is conjugated with glycine and taurine $[8,21]$. Gene expression of Baat, responsible for this conjugation, was not affected by KPH treatment compared to casein treated animals (Fig. $5 b)$. The gene expression of $F x r$ however, important in maintaining the BA homeostasis, was induced in KPH $(p=0,002)$ (Fig. 5c). 
a
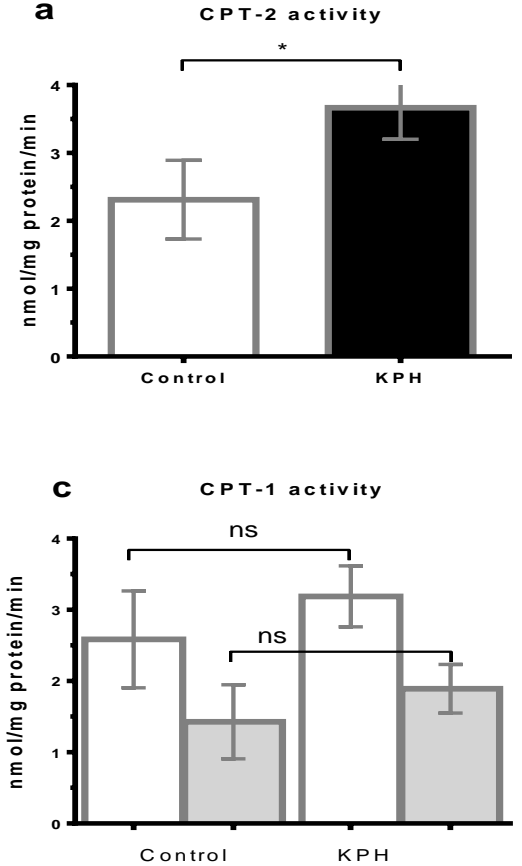

d

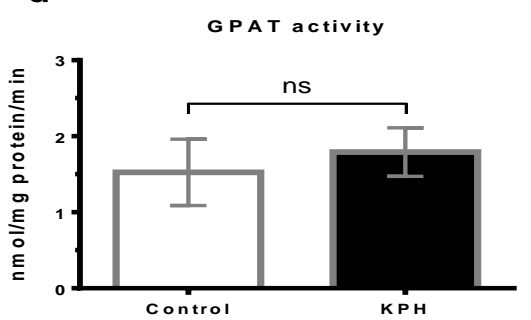

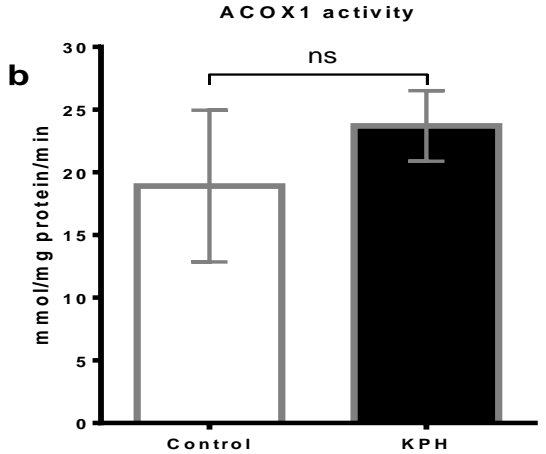

without MalonyI CoA

with MalonyI COA

$\mathbf{e}$

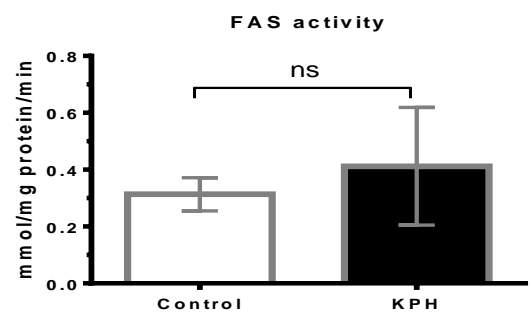

Figure 3. The effect of krill protein hydrolysate on hepatic enzyme activity

Hepatic activities of (a) CPT-2, (b) ACOX1, (c) CPT-1, (d) GPAT, and (e) FAS in male C57BL/6 mice fed high-fat diets containing casein or KPH. Data are presented as mean $\pm \mathrm{SD}, n=6$. $*$ Denote significant difference: Mann-Whitney test $(\mathrm{p}<0,05)$. ACOX1, acyl-CoA oxidase 1, palmitoyl; CPT-1, carnitine plamitoyltransferase 1; CPT-2, carnitine plamitoyltransferase 2; FAS, fatty acid synthase; GPAT, glycerol-3phosphate acyltransferase; KPH, krill protein hydrolysate; ns, non-significant.

The enterohepatic cycle begins at the canalicular membrane of the hepatocyte, where newly synthesized BAs are effluxed into the canaliculus. The gene expression of Bsep/Abcb1 1, responsible for this transport, was down-regulated by KPH feeding $(p=0,002)$ (Fig. 5d). Further, the expression of the cognate transporter $A b c b 4$, important for bile flow, was up-regulated by KPH feeding compared to the control ( $p=0,004)$ (Fig. 5e). The mRNA levels of $A b c c 2$ was also increased after KPH treatment $(p=0,02)$ (Fig. 5f), whereas the gene expression of $A b c b 1 b$ was unaffected (Fig. $5 \mathrm{~g}$ ). No differences in expression of the BA importer Slcolb2 were found (Fig. 5h), whereas the expression of Slc10al/Ntcp was significantly induced $(p=0,04)$ (Fig. 5i). Strikingly, the hepatic Abcc3 expression was induced 2.4 fold after KPH treatment $(p=0,02)$ (Fig. 5j), and the expression of Abcc4 almost 11-fold ( $p=0,002$ ) (Fig. 5k). 
Bile acid

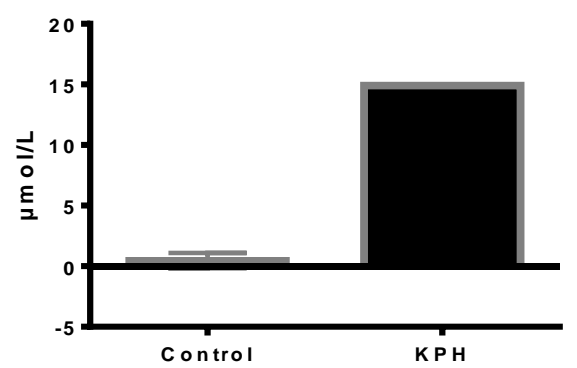

Figure 4. The effect of krill protein hydrolysate on plasma bile acid level

Concentrations of plasma bile acid (BA) in fasted male C57BL/6 mice fed high-fat diets containing casein or KPH. Data are analyzed of pooled samples of four animals. BA, bile acid; KPH, krill protein hydrolysate.
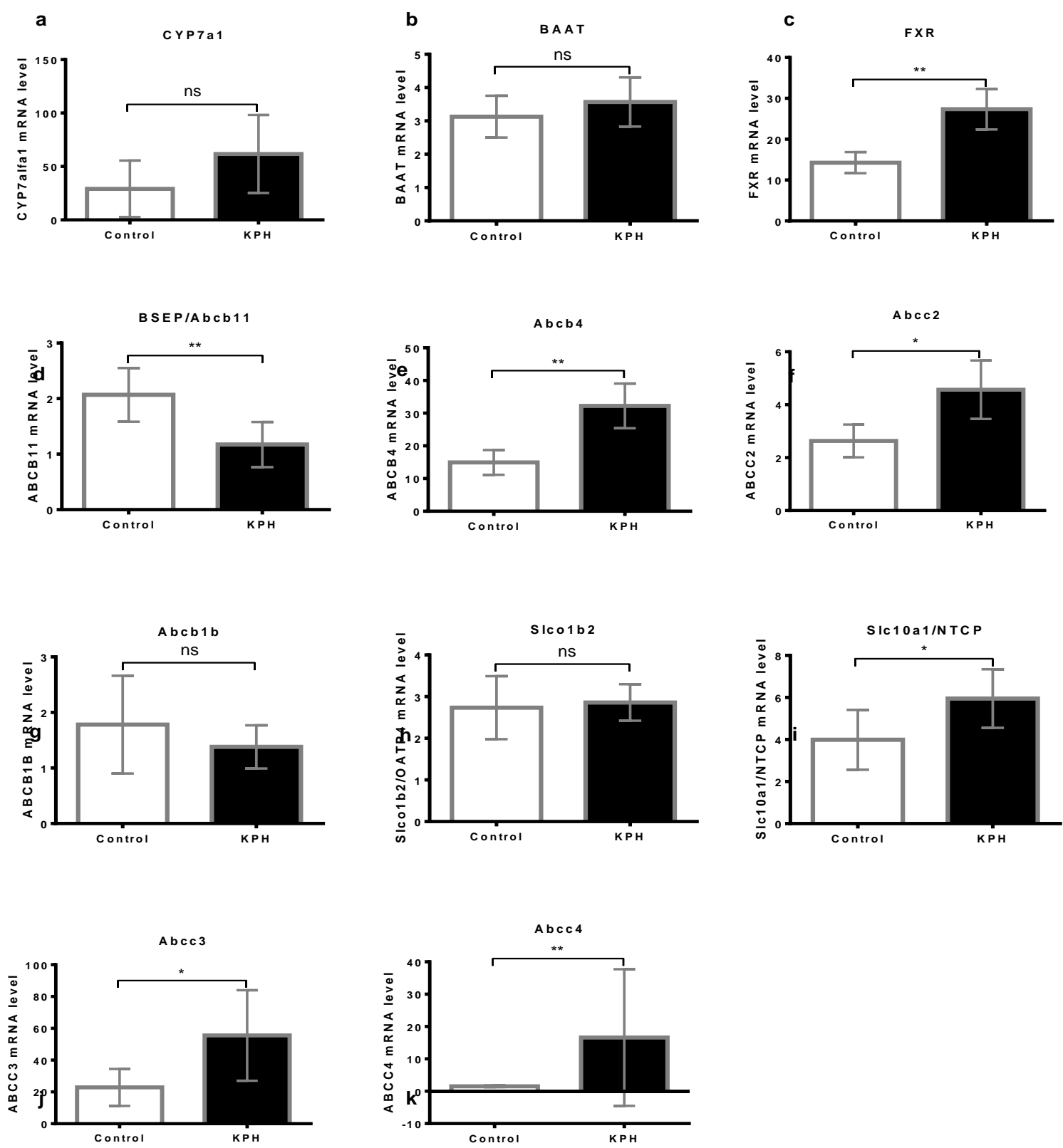

Figure 5. The effect of krill protein hydrolysate on expression of hepatic genes in the enterohepatic cycle. Hepatic gene expression of (a) Cyp7a1, the rate limiting enzyme in bile acid (BA) 
synthesis, (b) Baat, responsible for conjugating BAs to taurine and glycine, and of (c) Fxr, important in maintaining the BA homeostasis, in high-fat feeding containing casein or KPH. Gene expression of the canalucular exporters (d) Bsep/Abcb11, (e) Abcb4, (f) $A b c c 2$, (g) $A b c b 1 b$, the basolateral BA importers (h) Slcolb2, (i) Slc19a1/Ntcp, and the basolateral BA exporters (j) Abcc3 and (k) Abcc4. Values were related to the reference gene 18S. Data are presented as mean $\pm \mathrm{SD}, \mathrm{n}=6 . *$ Denote significant difference: Mann-Whitney test $\left.*^{*}=\mathrm{p}<0,05 ; * *=p<0,01\right)$. Abcb1b, ATP-binding cassette, sub-family B, member $1 \mathrm{~b}$; Abcb4, ATPbinding cassette sub-family $\mathrm{B}$, member 4 ; $\mathrm{ABCC} 2$, ATP-binding cassette sub-family $\mathrm{C}$, member 2; Abcc3, ATP-binding cassette, sub-family C, member 3; Abcc4, ATP-binding cassette, sub-family C, member 4; BA, bile acid; Baat, bile acid coenzymeA:aminoacid N-acyltransferase; Bsep/Abcb11, bile salt export pump; Cyp7a1, cytochrome P450, family 7, sub-family A, polypeptide 1; Fxr, farnesoid X receptor; Slco1b2, solute carrier organic anion transporter family, member 1b2; Slc10a1/Ntcp, solute carrier family 10, member 1.

\section{DISCUSSION}

In the present study we have shown that a protein hydrolysate from krill elevated BA level, with a concomitant reduction in plasma TAG concentration in high-fat fed C57BL/6 mice. Moreover, induction of enzymes involved in mitochondrial fatty acid oxidation and genes encoding proteins involved in BA synthesis, transport and secretion was found. Previously, we reported that krill powder, consisting of both protein and fatty acids, increases liver lipid catabolism in TNFa transgenic mice fed a high-fat diet [13]. These results are interesting, as it appears that the bioactive component in these two dietary supplementations lie in the protein fraction. The lipid lowering effect reported of KPH in the present study could be due to its amino acid composition. Studies have suggested that dietary proteins with low ratios of methionine/glycine and lysine/arginine have TAG-lowering effects [22]. As presented in table 2 , the amino acid composition in the two diets are different, and characterized by a lower ratio of methionine/glycine and lysine/arginine in the KPH than in the casein control.

Further, the data suggest that BA metabolism cross-talks with lipid metabolism. Indeed, BA administration has been shown to decrease plasma TAG concentration both in animals [1, 2] and humans with hypertriacylglycerolemia [23-25]. Further, increased concentration of BAs most plausible induces transcription of Fxr target genes [26, 27]. FXR is not only an important regulator for BA metabolism, but also for lipid metabolism [28]. FXR-deficient mice have increased synthesis of Apolipoprotein $\mathrm{B}(\mathrm{ApoB})$, resulting in higher TAG concentration in serum and liver [20]. It has also been reported that BA administration induces VLDL receptor transcription levels via a FXR-dependent mechanism [29], and this prevents elevated serum and liver TAG concentrations. Finally, BA treatment of mice seemed to down-regulate lipogenesis via repression of SREBP-1C [2].

We found no significant differences in hepatic activities of GPAT and FAS in KPHtreated mice compared to controls. Though, KPH treatment increased the hepatic fatty acid catabolism as the activity of the PPAR $\alpha$ target gene CPT-2 was increased. Further, the hepatic gene expression of Fxr was increased a 2-fold in the KPH treated mice compared to control. As FXR are reported to control plasma TAG levels by activating the PPAR $\alpha$ gene [3], and PPAR $\alpha$ is reported to induce genes encoding enzymes involved in mitochondrial fatty acid oxidation (reviewed in [4]), KPH regulation of BAs can possess the ability to modulate hepatic fatty acid oxidation, leading to reduced plasma TAG concentration. These results are in agreement with reported findings in rats by saithe Pollachius virens protein hydrolysate [30] and salmon protein hydrolysate [31].

The increased BA concentration could be due to different factors, including BA synthesis. Indeed, the gene expression of Cyp7al tended to be induced in KPH-treated mice. The gene expression of the BA export pump Bsep/Abcbll was down-regulated, and the 
phosphatidylcholine floppase Aacb4, which efflux the main components of bile, was upregulated after $\mathrm{KPH}$ treatment. The expression of the canalicular exporter $A b c b 1 b$ was however unchanged, whereas the expression of the BA exporter to the blood Abcc4 was induced by KPH feeding. Therefore, our data suggest that both higher BA synthesis and BA secretion to blood are underlying factors for the elevated plasma BA concentration.

\section{CONCLUSION}

Dietary treatment with KPH resulted in lower plasma concentration of TAGs and NEFAs, and elevated plasma level of BA compared to a casein control in high-fat fed mice. Further, induction of enzymes involved in mitochondrial fatty acid oxidation, and genes encoding proteins involved in BA synthesis, transport and secretion was found in the KPH-treated group. Altogether, it is plausible to suggest that lipid and BA metabolism can be modulated by protein hydrolysate derived from krill, and that increased mitochondrial fatty acid oxidation and elevated BA concentration regulates plasma levels of TAGs and NEFAs. It is unclear however, whether increased plasma BA levels are a cause or a response to hypotriacylglycerolemia.

\section{Abbreviations}

ABC (ATP-binding cassette), BA (bile acid), ACOX (acyl-CoA oxidase), CPT (carnitine palmitoyltransferase), CYP (cytochrome P450), FASN/FAS (fatty acid synthase), FXR (farnesoid X receptor), GPAT (glycerol-3-phosphate acyltransferase), KPH (krill protein hydrolysate), NEFA (non-esterified fatty acids), PPAR $\alpha$ (peroxisome proliferator-activated receptor alpha), SLC (solute carrier family), TAG (triacylglycerol), TNF $\alpha$ (tumor necrosis factor alpha), VLDL (very low density lipoprotein.

\section{Competing interests}

The authors declare that they have no competing interests.

\section{Author's contributions}

RKB and JS designed the research. BB, RKB and JS were involved in the acquisition of data. MSR, RV, BB, RKB analyzed and interpreted the data. MSR, BB, IB and RKB wrote the paper. All authors read and approved the final manuscript.

\section{Acknowledgments and Funding}

We thank to Kari Williams, Liv Kristine Øysæd, Randi Sandvik, Kari Helland Mortensen and Svein Krüger for technical assistance, and Eline Milde and staff at the Laboratory Animal Facility, University of Bergen, for care of the animals.

This project has been founded by NordForsk under the Nordic Centers of Excellence programme in Food, Nutrition, and Health, Project (070010), MitoHealth and Olympic Seafood AS. Olympic Seafood AS (Myrvaag, Norway) provided KPH, and Inge Bruheim and Marie Sannes Ramsvik are employed at Olympic Seafood AS.

\section{REFERENCES:}

1. Kast, H.R., et al., Farnesoid X-activated receptor induces apolipoprotein C-II transcription: a molecular mechanism linking plasma triglyceride levels to bile acids. Mol Endocrinol, 2001. 15(10): p. 1720-8. 
2. Watanabe, M., et al., Bile acids lower triglyceride levels via a pathway involving FXR, SHP, and SREBP-1c. J Clin Invest, 2004. 113(10): p. 1408-18.

3. Pineda Torra, I., et al., Bile acids induce the expression of the human peroxisome proliferator-activated receptor alpha gene via activation of the farnesoid $\mathrm{X}$ receptor. Mol Endocrinol, 2003. 17(2): p. 259-72.

4. Rakhshandehroo, M., et al., Peroxisome proliferator-activated receptor alpha target genes. PPAR Res, 2010. 2010.

5. Davidson, M.H., Mechanisms for the hypotriglyceridemic effect of marine omega-3 fatty acids. Am J Cardiol, 2006. 98(4A): p. 27i-33i.

6. Schmidt, S., et al., Regulation of lipid metabolism-related gene expression in whole blood cells of normo- and dyslipidemic men after fish oil supplementation. Lipids Health Dis, 2012. 11: p. 172.

7. Staels, B. and V.A. Fonseca, Bile acids and metabolic regulation: mechanisms and clinical responses to bile acid sequestration. Diabetes Care, 2009. 32 Suppl 2: p. S237-45.

8. Lefebvre, P., et al., Role of bile acids and bile acid receptors in metabolic regulation. Physiol Rev, 2009. 89(1): p. 147-91.

9. Kersten, S., et al., The peroxisome proliferator-activated receptor alpha regulates amino acid metabolism. FASEB J, 2001. 15(11): p. 1971-8.

10. Sheikh, K., et al., Beyond lipids, pharmacological PPARalpha activation has important effects on amino acid metabolism as studied in the rat. Am J Physiol Endocrinol Metab, 2007. 292(4): p. E1157-65.

11. Batetta, B., et al., Endocannabinoids may mediate the ability of (n-3) fatty acids to reduce ectopic fat and inflammatory mediators in obese Zucker rats. J Nutr, 2009. 139(8): p. 1495-501.

12. Tandy, S., et al., Dietary krill oil supplementation reduces hepatic steatosis, glycemia, and hypercholesterolemia in high-fat-fed mice. J Agric Food Chem, 2009. 57(19): p. 9339-45.

13. Bjorndal, B., et al., Krill powder increases liver lipid catabolism and reduces glucose mobilization in tumor necrosis factor-alpha transgenic mice fed a high-fat diet. Metabolism, 2012. 61(10): p. 1461-72.

14. Hatanaka, A., et al., Isolation and identification of antihypertensive peptides from antarctic krill tail meat hydrolysate. J Food Sci, 2009. 74(4): p. H116-20.

15. Yamada, H., T. Ueda, and A. Yano, Water-soluble extract of Pacific Krill prevents triglyceride accumulation in adipocytes by suppressing PPARgamma and C/EBPalpha expression. PLoS One, 2011. 6(7): p. e21952.

16. Bligh, E.G. and W.J. Dyer, A rapid method of total lipid extraction and purification. Can J Biochem Physiol, 1959. 37(8): p. 911-7.

17. Berge, R.K., T. Flatmark, and H. Osmundsen, Enhancement of long-chain acyl-CoA hydrolase activity in peroxisomes and mitochondria of rat liver by peroxisomal proliferators. Eur J Biochem, 1984. 141(3): p. 637-44.

18. Skorve, J., et al., On the mechanism of the hypolipidemic effect of sulfur-substituted hexadecanedioic acid (3-thiadicarboxylic acid) in normolipidemic rats. J Lipid Res, 1993. 34(7): p. 1177-85. 
19. Vigerust, N.F., et al., Fish oil and 3-thia fatty acid have additive effects on lipid metabolism but antagonistic effects on oxidative damage when fed to rats for 50 weeks. J Nutr Biochem, 2012. 23(11): p. 1384-93.

20. Lambert, G., et al., The farnesoid X-receptor is an essential regulator of cholesterol homeostasis. J Biol Chem, 2003. 278(4): p. 2563-70.

21. Shonsey, E.M., et al., Bile acid coenzyme A: amino acid N-acyltransferase in the amino acid conjugation of bile acids. Methods Enzymol, 2005. 400: p. 374-94.

22. Kritchevsky, D., et al., Atherogenicity of animal and vegetable protein. Influence of the lysine to arginine ratio. Atherosclerosis, 1982. 41(2-3): p. 429-31.

23. Angelin, B., et al., Effects of cholestyramine and chenodeoxycholic acid on the metabolism of endogenous triglyceride in hyperlipoproteinemia. J Lipid Res, 1978. 19(8): p. 1017-24.

24. Bateson, M.C., et al., Chenodeoxycholic acid therapy for hypertriglyceridaemia in men. Br J Clin Pharmacol, 1978. 5(3): p. 249-54.

25. Carulli, N., et al., Chenodeoxycholic acid and ursodeoxycholic acid effects in endogenous hypertriglyceridemias. A controlled double-blind trial. J Clin Pharmacol, 1981. 21(10): p. 436-42.

26. Parks, D.J., et al., Bile acids: natural ligands for an orphan nuclear receptor. Science, 1999. 284(5418): p. 1365-8.

27. Makishima, M., et al., Identification of a nuclear receptor for bile acids. Science, 1999. 284(5418): p. 1362-5.

28. Claudel, T., B. Staels, and F. Kuipers, The Farnesoid X receptor: a molecular link between bile acid and lipid and glucose metabolism. Arterioscler Thromb Vasc Biol, 2005. 25(10): p. 2020-30.

29. Sirvent, A., et al., The farnesoid $X$ receptor induces very low density lipoprotein receptor gene expression. FEBS Lett, 2004. 566(1-3): p. 173-7.

30. Liaset, B., et al., Fish protein hydrolysate elevates plasma bile acids and reduces visceral adipose tissue mass in rats. Biochim Biophys Acta, 2009. 1791(4): p. 254-62.

31. Wergedahl, H., et al., Fish protein hydrolysate reduces plasma total cholesterol, increases the proportion of HDL cholesterol, and lowers acyl-CoA:cholesterol acyltransferase activity in liver of Zucker rats. J Nutr, 2004. 134(6): p. 1320-7. 